\begin{tabular}{|c|c|c|}
\hline $\begin{array}{l}\text { ISSN 2525-4812 (versão online) } \\
\text { ISSN 2238-7641 (versão impressa) } \\
\text { http://www.revistaterceiramargem.com/ } \\
\text { index.php/terceiramargem/index }\end{array}$ & $\begin{array}{c}\text { Recebido em: 30/8/2019 } \\
\text { Aprovado em: 6/1/ } 2020 \\
\text { Período de publicação: jan., } 2021\end{array}$ & $\begin{array}{c}\text { Revista Terceira } \\
\text { Margem Amazônia } \\
(\text { v. } 6 \cdot \text { n. especial } 16 \cdot \text { Jan. } 2021)\end{array}$ \\
\hline
\end{tabular}

Como citar o artigo:

BATISTA, A. W. S.; Variação intraespecífica de pitaica (Swartzia polyphylla DC.) (Fabaceae) em florestas inundáveis, Caxiuanã, Pará. Revista Terceira Margem Amazônia. v. 6, n. especial 16, p. 191-207, 2021. DOI: http://dx.doi.org/10.36882/2525-4812. 2021v6i16.ed.esp.p191-207

\title{
VARIAÇÃO INTRAESPECÍFICA DE PITAICA (Swartzia polyphylla DC.) (Fabaceae) EM FLORESTAS INUNDÁVEIS, CAXIUANÃ, PARÁ
}

\author{
Annanda Waneza da Silva Batista \\ Markus Meireles Campos ${ }^{2}$ \\ Renan Cunha Ribeiro ${ }^{3}$
}

\begin{abstract}
Resumo: O estudo investigou se houve diferença na densidade de indivíduos, nos atributos funcionais foliares, na germinação e no crescimento das plântulas de pitaica (Swartzia polyphylla DC.) (Fabaceae) em duas florestas inundadas (igapó e várzea) da Estação Científica Ferreira Penna, em Caxiuanã, no estado do Pará. Houve diferenças significativas na densidade de plântulas e de plantas estabelecidas de pitaica (S. polyphylla) entre os dois tipos de floresta. Nos traços funcionais, houve diferença significativa na área foliar (LA) e no conteúdo de matéria seca foliar (LDMC), assim como no peso fresco e seco, comprimento e espessuras das sementes. Houve também diferença significativa na taxa de germinação das sementes entre os ambientes. As curvas de crescimento das sementes foram semelhantes entre as florestas. Os resultados inferem que estratégias para o estabelecimento de sementes podem estar ligadas ao investimento em atributos funcionais diferentes nos ambientes (área foliar, área foliar específica e conteúdo de matéria seca foliar), já que a folha, por possuir todo o aparato fotossintético da planta, é o órgão que mais responde de forma plástica à variabilidade de condições ambientais.
\end{abstract}

Palavras-chave: Amazônia, sementes, germinação, traços funcionais.

\section{INTRASPECIFIC VARIATION OF PITAICA (Swartzia polyphylla DC.) (Fabaceae) IN FLOODED FORESTS, CAXIUANÃ, PARÁ}

\footnotetext{
Bióloga, M. Sc. em Botânica Tropical, Belém, PA. E-mail: annandawaneza@yahoo.com.br

(D) https://orcid.org/0000-0002-6031-8817

2 Biólogo, mestrando em Ciências Biológicas, Belém, PA. E-mail: markus_mmc@yahoo.com.br

(iD https://orcid.org/0000-0002-1390-2754

3 Biólogo, Belém, PA. E-mail: renancunhaufpa@yahoo.com.br

(D) https://orcid.org/0000-0003-1840-2983
}

Abstract: The study investigated whether there was a difference in density of individuals, foliar functional attributes, germination and seedling growth of Swartzia polyphylla DC. (Fabaceae) in two flooded forests (igapó and varzea) of the Ferreira Penna Scientific Station, in Caxiuanã, Pará State. There were significant differences in the density of established plantlets of $S$. polyphylla between the two types of forests. In the functional traits, there was a significant difference in leaf area (LA) and dry matter content (LDMC). The- 
re was significant difference in fresh and dry weight, length and thickness of the seeds. There was also a significant difference in seed germination rate between the environments. Growth curves of S. polyphylla seeds were similar between the two types of forest. The results allow to infer that strategies for the establishment of seeds can be linked to the investment in different functional attributes in the environments (leaf area, specific leaf area and leaf dry matter content), since the leaf, having all the photosynthetic apparatus of the plant, is the organ that responds most morphologically to the variability of environmental conditions.

Keywords: Amazon, flooded forests, seeds, germination. functional traits.

\section{Introdução}

A região amazônica abrange a maior floresta tropical do planeta, com grande variedade de ecossistemas e alta diversidade biológica (BRASIL, 2002). Dentre os ecossistemas presentes nessa floresta destacam-se as áreas inundáveis, que são de extrema importância, devido a sua grande interação com ecossistemas aquáticos, abrigando não somente espécies endêmicas, como também sustentando muitas plantas e insetos que mantêm e formam a base das cadeias alimentares (GOULDING, 1980; SIOLI, 1991). Nesses ambientes estão inseridas a Floresta Ombrófila Densa Aluvial e Ombrófila Aberta Aluvial, ou popularmente chamadas de várzeas e igapós, respectivamente (MANUAL..., 2012).

As várzeas são planícies periodicamente inundáveis por rios de água branca ou barrenta, provenientes das áreas andina e pré-andina. Muito ricas em nutrientes, ocupam a maioria das áreas inundáveis da Floresta Amazônica, possuindo grande fertilidade em seus solos, devido à renovação anual da deposição de nutrientes carreados pelas enchentes e pela formação recente de seus solos, que datam o período quaternário (JUNK, 1984; SIOLI, 1991; AYRES, 2006).

Os igapós, por outro lado, são planícies periodicamente inundáveis por rios de águas pretas ou claras que nascem nos escudos arqueanos das Guianas e do Brasil Central. A formação antiga de seus solos, por rochas do Período Pré-Cambriano a Terciário, permite aos rios originários nessas regiões o transporte de poucos sedimentos em suspensão, isso confere transparência às suas águas, que são pobres em nutrientes e muito ácidas devido à grande quantidade de matéria orgânica decomposta e à produção de ácidos fúlvicos e húmicos (SIOLI, 1991; JUNK, 1993, 1997; AYRES, 2006).

Devido às oscilações sazonais no nível das águas, a maioria das espécies arbóreas das florestas inundáveis amazônicas sincroniza a floração e a frutificação, de modo que ocorram em concomitância com o ciclo hidrológico. Assim, a maturação dos frutos geralmente ocorre nos períodos de pico da inundação, onde a superfície é coberta por água e invadida por peixes, facilitando a dispersão das sementes (KUBTZKI; ZIBURSKI, 1994; PAROLIN, 2002). Depois de cair na água, os diásporos são sujeitos a períodos variáveis de flutuabilidade e/ou submersão. As sementes que flutuam podem permanecer nessas condições durante períodos de até 2 meses (KUBITZKI, 1985); para isso possuem tecidos esponjosos ou grandes espaços cheios de ar que aumentam a flutuação (KUBITZKI; ZIBURSKI, 1994; WILLIAMSON et al., 1999). 
As sementes podem passar longos períodos submersas e permanecerem viáveis até que as condições ambientais se tornem adequadas para que elas possam germinar e formar uma plântula, geralmente no início da fase terrestre. Entretanto, alguns estudos têm demostrado que a saturação de água do solo não impede que algumas plantas germinem suas sementes e produzam plântulas enquanto ainda estão boiando ou mesmo submersas (PAROLIN; JUNK, 2002; SCARANO et al., 2003; FERREIRA et al., 2007; WITTMANN et al., 2007), indicando que as plântulas de algumas espécies são altamente tolerantes a inundação, podendo sobreviver por vários meses nessas condições (PAROLIN, 2002; PAROLIN; JUNK, 2002; FERREIRA et al., 2007, 2009). O grau de tolerância de cada espécie varia de acordo com a sua posição no gradiente de inundação. A vegetação que ocupa as cotas mais baixas está mais bem adaptada a essas condições, em relação àquela que ocupa cotas mais altas (WITTMANN et al., 2002; PAROLIN et al., 2003).

A espécie pitaica (S. polyphylla DC.) (Fabaceae) é uma árvore de porte mediano a grande, atingindo até 40 metros de altura, cujo tronco pode chegar a $4 \mathrm{~m}$ de diâmetro. Geralmente apresenta sapopemas altas e onduladas, com exsudação vermelha abundante na entrecasca. Os folíolos são também distintos por causa das nervuras impressas na superfície adaxial. De acordo com Cowan (1967) e Ribeiro et al., (1999), sua distribuição geográfica vai da Amazônia Central até as Guianas, sendo encontrada na Floresta Amazônica em áreas de várzea e igapó.

Além do grande potencial madeireiro que essa espécie possui, várias substâncias como flavonoides, saponinas e diterpenoides, obtidas de seus frutos, folhas, caule e sementes, vêm sendo testadas para o controle de fungos, bactérias, insetos e de moluscos causadores de doenças, evidenciando assim sua grande importância farmacológica (SANCHEZ et al., 1999; REICH et al., 2003; ARAÚJO et al., 2009).

A distribuição dos organismos, segundo a ecologia funcional de plantas, não acontece por acaso, havendo, portanto, uma relação entre as diferenças funcionais das plantas e sua distribuição em diferentes habitats (DUARTE, 1999). Essa abordagem baseia-se na teoria de diferenciação de nichos (MACARTHUR; LEVINS, 1967; MAY, 1972), a qual considera as espécies como entidades heterogêneas que respondem diferentemente às condições ambientais. Para essa teoria, a diferenciação das espécies, independentemente de classificação taxonômica, ocorre com a variabilidade dos traços funcionais (VIOLLE; JIANG, 2009) a partir de filtros ambientais (VIOLLE et al., 2007).

Dentre os traços mais utilizados na ecologia vegetal têm-se os traços funcionais foliares (área foliar, área foliar específica e conteúdo de matéria seca foliar), já que a folha, por possuir todo o aparato fotossintético da planta, é o órgão que mais responde de forma plástica à variabilidade de condições ambientais (REICH et al., 2003; MONTEIRO et al., 2005; SOUZA et al., 2015).

Existem poucos estudos sobre fisiologia da germinação e estabelecimento de plântulas em florestas de inundação (SCARANO et al., 1998; PAROLIN, 2001; PAROLIN; JUNK, 2002; FERREIRA et al., 2007; SWENSON; ENQUIST, 2007; WITTMANN et al., 2007), apesar da grande importância das áreas inundáveis da Amazônia. Além disso, a proposta de ecologia baseada em traços funcionais vem crescendo consideravelmente (ACKERLY; CORNWELL, 2007; SWENSON; ENQUIST, 2007; KRAFT et al., 2008; CORNWELL; ACKERLY, 2009). 
Diante do que foi exposto, o estudo com a espécie pitaica (S. polyphylla) mostra-se importante, pois permite averiguar como ela sobrevive em ambientes tão distintos, como as florestas de várzea e igapó. O objetivo do estudo foi analisar a densidade de indivíduos da população da regeneração natural e plantas estabelecidas com diâmetro de até $10 \mathrm{~cm}$, atributos funcionais foliares, caracteres biométricos e germinação de sementes e crescimento de plântulas de pitaica (S. polyphylla) em florestas de várzea e igapó da Estação Científica Ferreira Penna (ECFPn) na Amazônia Oriental. As hipóteses propostas foram: 1) existe diferença na densidade de indivíduos da população e nos atributos funcionais foliares de pitaica (S. polyphylla) entre as florestas de igapó e várzea; e 2) existe diferença na germinação de sementes e crescimento das plântulas de pitaica (S. polyphylla) entre as florestas de igapó e várzea.

\section{Materiais e Métodos}

O estudo foi realizado nas florestas de igapó e de várzea, localizadas na Estação Científica Ferreira Penna (ECFPn), a qual se encontra inserida na Floresta Nacional (Flona) de Caxiuanã, criada em 28 de novembro de 1961, sendo uma das mais antigas unidades de conservação federal existentes no Pará. Possui uma área de aproximadamente 330 mil hectares e está situada às margens da Baía de Caxiuanã e dos Botos, entre os rios Xingu e Anapu, nos municípios de Portel e Melgaço, no estado do Pará.

O Plano de Manejo da Flona definiu como Zona de Manejo Florestal Sustentável 183.695,7 ha, dos quais 176 mil foram destinados à concessão florestal. A área para concessão foi dividida em três unidades de manejo florestal (UMF): a UMF I de 37.365 ha, a UMF II de 87.067 ha e a UMF III, de 52.168 ha (Figura 1).

Figura 1. Localização da Floresta Nacional de Caxiuanã no estado do Pará e nos municípios.

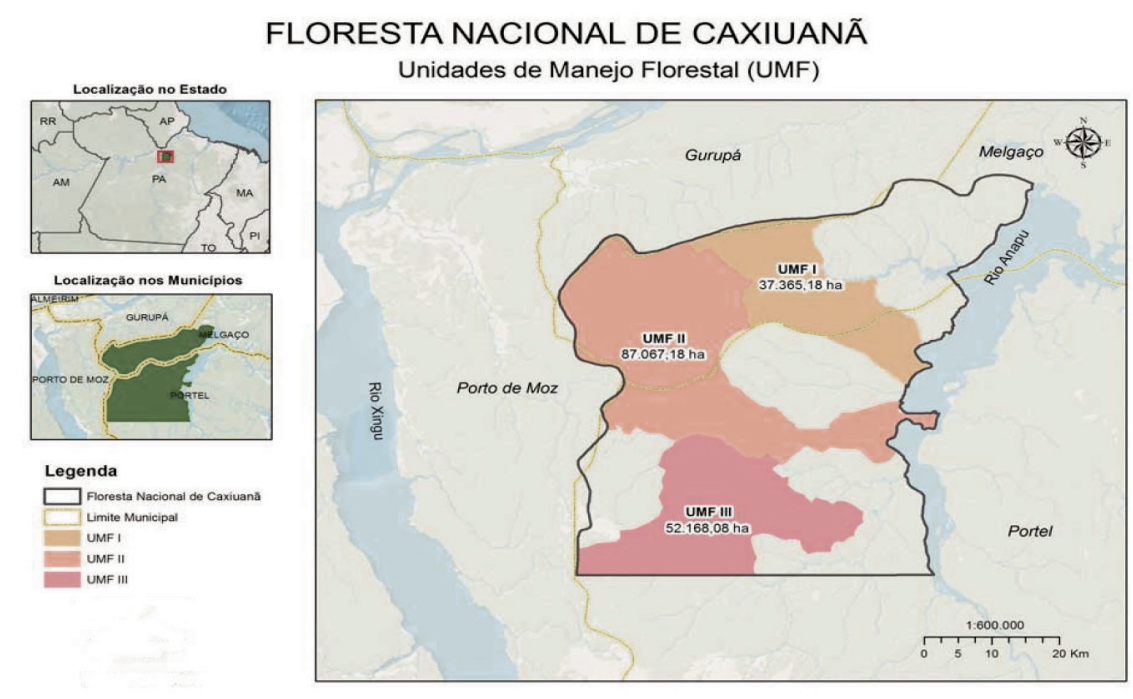

Fonte: Serviço Florestal Brasileiro (2019).

A cobertura vegetal da Flona é bem diversificada, apresenta Floresta Ombrófila Densa das Terras Baixas (platôs e baixios), denominada localmente de terra firme, com predominância de $85 \%$ da área total da vegetação; florestas secundárias de diferentes idades; campinaranas e encra- 
ves de vegetação de savana, correspondendo a 5\% da cobertura vegetal e as Florestas Ombrófilas Aluviais que ocupam os 10\% restantes (FERREIRA et al., 2012).

Para a estrutura das populações realizou-se levantamento botânico em 2014 usando 15 parcelas na floresta de igapó do Rio Caxiuanã e 15 parcelas na floresta de várzea na Baía de Caxiuanã. A distância entre as parcelas variou de $800 \mathrm{~m}$ a $30 \mathrm{~km}$. Houve a divisão em dois estratos: 1) regeneração natural (indivíduos com até $30 \mathrm{~cm}$ de altura) usando 15 parcelas $2 \times 2$ metros; e 2) plantas estabelecidas (diâmetros menores que $10 \mathrm{~cm}$ ) usando 15 parcelas de 5 x 5 metros. Nas parcelas de plantas estabelecidas, todos os indivíduos de pitaica (S. polyphylla) foram contados, medindo-se o diâmetro, enquanto nas parcelas da regeneração, todos os indivíduos foram contados.

As folhas de pitaica (S. polyphylla) nas florestas de igapó e várzea foram coletadas às margens do Rio Curuá e na Baía de Caxiuanã, respectivamente. Foram selecionadas aleatoriamente dez árvores em cada floresta, com DAP $\geq 10 \mathrm{~cm}$ e que se encontravam distantes pelo menos $1 \mathrm{~km}$ uma das outras, para reduzir a probabilidade de coletar indivíduos geneticamente semelhantes (HULSHOF; SWENSON, 2010). De cada árvore foram coletadas cinco amostras de folhas relativamente jovens (presumivelmente mais fotossinteticamente ativas), mas totalmente expandidas e endurecidas, não danificadas por ataque de patógenos ou herbívoros e que estavam expostas ao sol ou em partes menos sombreadas.

A coleta foi realizada com o auxílio de um podão de $5 \mathrm{~m}$. As folhas foram embrulhadas em papel úmido e colocadas em sacos plásticos. Em laboratório, as folhas foram pesadas utilizando-se uma balança digital de precisão para a obtenção da massa fresca foliar (FLM). Posteriormente, as folhas foram digitalizadas em scanner de mesa, para subsequentes análises da área foliar (LA). A área foliar foi calculada com auxílio do programa ImageJ versão 1.44p (RASBAND, 2016). Após esse procedimento as folhas foram colocadas para secar em estufa a $60^{\circ} \mathrm{C}$ durante 72 horas.

Ao final foram medidos quatro traços funcionais que estão relacionados aos recursos do solo, à força competitiva e à captação de luz, segundo o protocolo de Pérez-Harguindeguy et al. (2013), os quais são: área foliar (LA), área foliar específica (SLA), conteúdo de matéria seca das folhas (LDMC) e massa seca por área foliar (LMA). Os traços foliares foram calculados considerando a folha como um todo (pecíolo, raquis foliar e folíolos), e não entre folíolos, pois como se trata de folhas compostas a variabilidade entre os folíolos seria muito maior (HULSHOF; SWENSON, 2010; PRADO JÚNIOR et al., 2014).

As sementes dos espécimes de pitaica (S. polyphylla) nas florestas de igapó e várzea foram coletadas às margens do Rio Curuá e da Baía de Caxiuanã, em pontos extremos. Foram selecionadas as que se encontravam no solo ou boiando na água próximo das árvores-mãe, sendo excluídas sementes quebradas, trincadas e furadas. De dez árvores em cada local, coletou-se um total de 118 sementes no igapó e 130 na várzea. Escolheu-se aleatoriamente 30 sementes oriundas da floresta de igapó e 30 da floresta de várzea para baldes com água até serem semeadas. O restante (88 sementes de igapó e 100 de várzea) foi utilizado para as medições biométricas (comprimento, espessura, peso seco do tegumento e peso fresco e seco da semente). As sementes foram plantadas individualmente em recipientes plásticos, com dimensões de $36 \mathrm{~cm}$ x $5 \mathrm{~cm}$ de profundidade e identificadas com placas de alumínio a que floresta pertenciam. 
O experimento contou com um total de 60 sementes, sendo 30 de igapó e 30 de várzea. Após o plantio, ele foi acompanhado diariamente, e as sementes foram irrigadas manualmente em dias alternados. Consideraram-se germinadas as sementes que apresentaram ruptura do tegumento (PELAZZA et al., 2011).

\section{Análises de dados}

\section{Estrutura da população}

As diferenças das médias do número de indivíduos da regeneração natural e plantas estabelecidas entre os tipos de vegetação foram comparadas usando o Teste t para amostras independentes ou com o Teste U-Mann-Whitney quando a homogeneidade dos dados não cumpria com os pressupostos das análises paramétricas (ZAR, 2010).

Os traços funcionais foliares em relação aos tipos de vegetação foram testados com Teste $\mathrm{t}$ de Student e quando a homogeneidade dos dados não cumpria com os pressupostos das análises paramétricas foi usado o teste não paramétrico Teste U-Mann-Whitney (ZAR, 2010).

Para analisar a correlação entre os atributos funcionais para as florestas alagadas foi realizada uma Análise de Componentes Principais (PCA) usando o programa PCORD (Multivariate Analysis of Ecological Data Version 6, https://www.pcord.com/pc6fixes.htm)

As diferenças dos caracteres biométricos das sementes de pitaica ( $S$. polyphylla) entre os tipos de vegetação foram comparadas usando o Teste t para amostras independentes ou o Teste U-Mann-Whitney quando a homogeneidade dos dados não cumpria com os pressupostos das análises paramétricas (ZAR, 2010). Para calcular as taxas de germinação, as sementes dos recipientes plásticos foram agrupadas em seis blocos, contendo cinco sementes em cada bloco.

\section{Resultados e Discussão}

\section{Estrutura da população}

Houve diferenças significativas da densidade de plântulas e de plantas estabelecidas de pitaica (S. polyphylla) entre os dois tipos de florestas inundadas, sendo estas maiores nas parcelas de floresta de igapó em comparação a floresta de várzea (Figura 2).

Na floresta de várzea, a maior disponibilidade nutricional do solo e altura do terreno promovem maior investimento dos indivíduos em atributos funcionais que garantam rápida aquisição de recursos e crescimento, como estratégia para "fugir do alagamento" e da forte concorrência interespecífica por esses recursos (PAROLIN, 2002). Por outro lado, os indivíduos que vivem no igapó, ambiente nutricionalmente pobre e de baixo relevo, preferem investir em atributos que promovam a retenção e conservação de recursos, apresentando menores taxas de crescimento, porém maior capacidade de sobrevivência em condições de estresse (WRIGHT et al., 2010; PHILIPSON et al., 2014). Essa estratégia pode ter contribuído para a maior densidade de indivíduos de pitaica (S. polyphylla) nas florestas de igapó em comparação às de várzea. 
Figura 2. Média do número de plântulas (a) e de plantas estabelecidas (b) de Swartzia polyphylla entre as florestas de igapó e várzea.

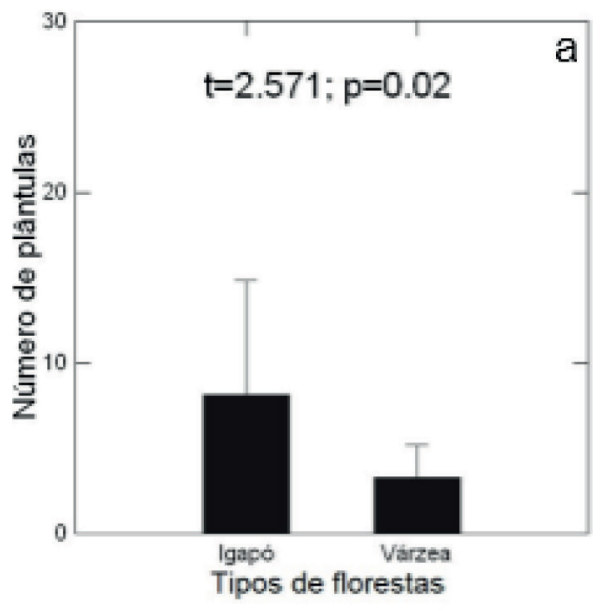

Fonte: Elaborado pelos autores (2017)

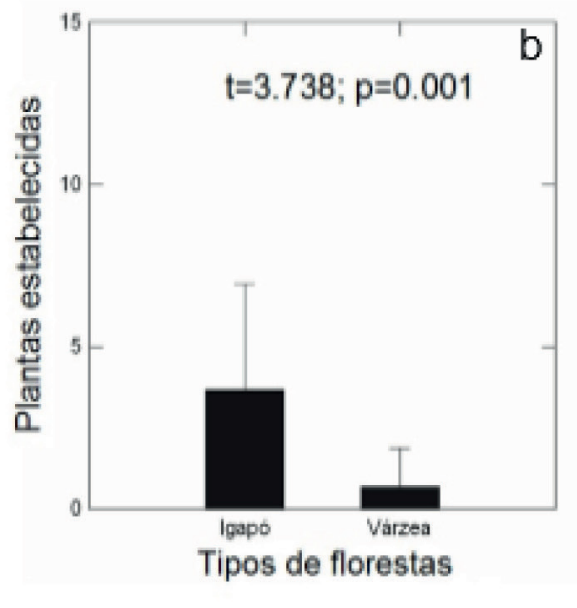

Os diásporos oriundos da várzea têm peso maior que os encontrados no igapó, de acordo com o presente estudo. Trabalhos relativamente recentes mostram que a massa da semente pode ser considerada um atributo importante para a germinação, desenvolvimento e estabelecimento da plântula (LEISHMAN et al., 2000; MELO et al., 2004; NORDEN et al., 2009; HENNING et al., 2010). A quantidade de massa da semente tem forte influência ambiental (MICHAELS et al., 1988; WESTOBY et al., 1992); e como várzea e igapó são ambientes distintos, espera-se que as sementes sofram diferentes pressões seletivas em relação ao seu peso e tamanho.

Além do peso maior nas sementes de várzea foram encontrados valores maiores no tamanho das sementes e espessura delas, podendo explicitar diferenças na disponibilidade nutricional, no modo de dispersão e condições de estabelecimento (WESTOBY et al., 1992; PAROLIN, 2000; FENNER; THOMPSON, 2005; SORIANO et al., 2011). Sendo assim, as sementes maiores encontradas na várzea podem estar associadas à riqueza de nutrientes presente no solo dessa floresta, o que possibilita às sementes a produção de mais endosperma e reservas energéticas para o embrião em desenvolvimento (MICHAELS et al., 1988; MOEGENBURG, 1996). Por outro lado, no igapó, a presença de sementes menores pode refletir uma estratégia de retenção e conservação de recursos, principalmente por se tratar de um ambiente pobre em nutrientes. Além disso, o tamanho e o peso seco menor nas sementes de igapó podem permitir que elas ocupem maior número de micro-habitats (JORDANO, 2000) devido a maior facilidade de serem ingeridas por peixes, ou mesmo transportadas por vetores como o vento e a água (MOEGENBURG, 2002), principais dispersores nas áreas alagáveis (KUBITZKI; ZIBURSKI, 1994). Isso reafirma a maior densidade de plântulas e plantas adultas encontradas no igapó.

\section{Traços Funcionais Foliares}

A medição dos traços foliares de pitaica (S. polyphylla) apresentou diferenças significativas na área foliar (LA) e no conteúdo de matéria seca foliar (LDMC) entre as florestas de igapó e várzea; com relação à área foliar específica (SLA) e à massa seca por área foliar (LMA) não foram observadas diferenças significativas entre os ambientes (Tabela 1). 
Tabela 1. Traços funcionais e seus valores estatísticos.

\begin{tabular}{|ccccc|} 
Traço funcional & $\begin{array}{c}\text { Média e desvio } \\
\text { padrão várzea }\end{array}$ & $\begin{array}{c}\text { Média e desvio } \\
\text { padrão igapó }\end{array}$ & $\mathbf{t}$ & $\mathbf{p}$ \\
LA & $549,7(107,2)$ & $429,6(109,07)$ & $-2,483$ & 0,023 \\
SLA & $149,6(25,9)$ & $178,8(58,9)$ & 1,436 & 0,176 \\
LDMC & $0,499(0,019)$ & $0,426(0,049)$ & $-4,432$ & 0,001 \\
LMA & $0,007(0,001)$ & $0,006(0,002)$ & $-1,165$ & 0,263 \\
\hline
\end{tabular}

Fonte: Elaborado pelos autores (2017).

A diferença significativa na área foliar (LA) entre as florestas está de acordo com as afirmações de Klinge et al. (1983), que verificaram a presença de folhas do tipo mesófila nas várzeas e notófilas (folhas menores) no igapó. A área foliar maior na várzea pode estar relacionada com a maior riqueza de nutrientes presente em seu solo, já que uma folha maior demanda um custo energético e nutricional também maior (PICCININ; RUIVO, 2012). Além disso, essa grande disponibilidade de nutrientes também pode ter promovido um LDMC maior na várzea, ou seja, um maior investimento na dureza, resistência e produtividade da folha (PÉREZ-HARQUINDEGUY et al., 2013).

Com relação à área foliar específica (SLA), autores indicam que altos valores de SLA estão associados a maiores taxas de crescimento vegetal e folhas pouco longevas; por outro, espécies com SLA reduzido apresentam folhas com maior longevidade e alta resistência estrutural contra herbívoros, porém menor crescimento devido a sua baixa eficiência fotossintética (REICH et al., 1999; WRIGHT; CANNON, 2001; POORTER; BONGERS, 2006). Esse traço é o inverso do LMA, assim folhas com alto SLA geralmente possuem baixo LMA (REICH et al., 1997; POORTER et al., 2009). Nesse sentido, a falta de diferença significativa entre os ambientes com relação aos dois traços sugere que a capacidade fotossintética por unidade de massa, taxa de crescimento relativo e longevidade da folha de pitaica ( $S$. polyphylla) provavelmente é semelhante em ambas as florestas.

\section{Distribuição dos traços funcionais}

A distribuição dos traços funcionais nas populações de pitaica (S. polyphylla) foram diferentes entre os dois tipos de floresta (Figura 3).

Distintas estratégias têm sido verificadas para as espécies que colonizam esses ambientes, relacionadas com local em que a planta está estabelecida no gradiente do solo e a cota de inundação a qual é submetida. Com isso a variação na distribuição dos traços funcionais entre as espécies nos dois locais indica diversificação de estratégias na aquisição dos recursos disponíveis (JUNK, 1989; WITTMANN et al., 2002; PAROLIN et al., 2004). 
Figura 3. Resultado da análise de componentes principais (PCA) dos atributos funcionais foliares de Swartzia polyphylla DC entre as florestas da Estação Científica Ferreira Penna, Caxiuanã, Pará (i=Igapó; v=Várzea).

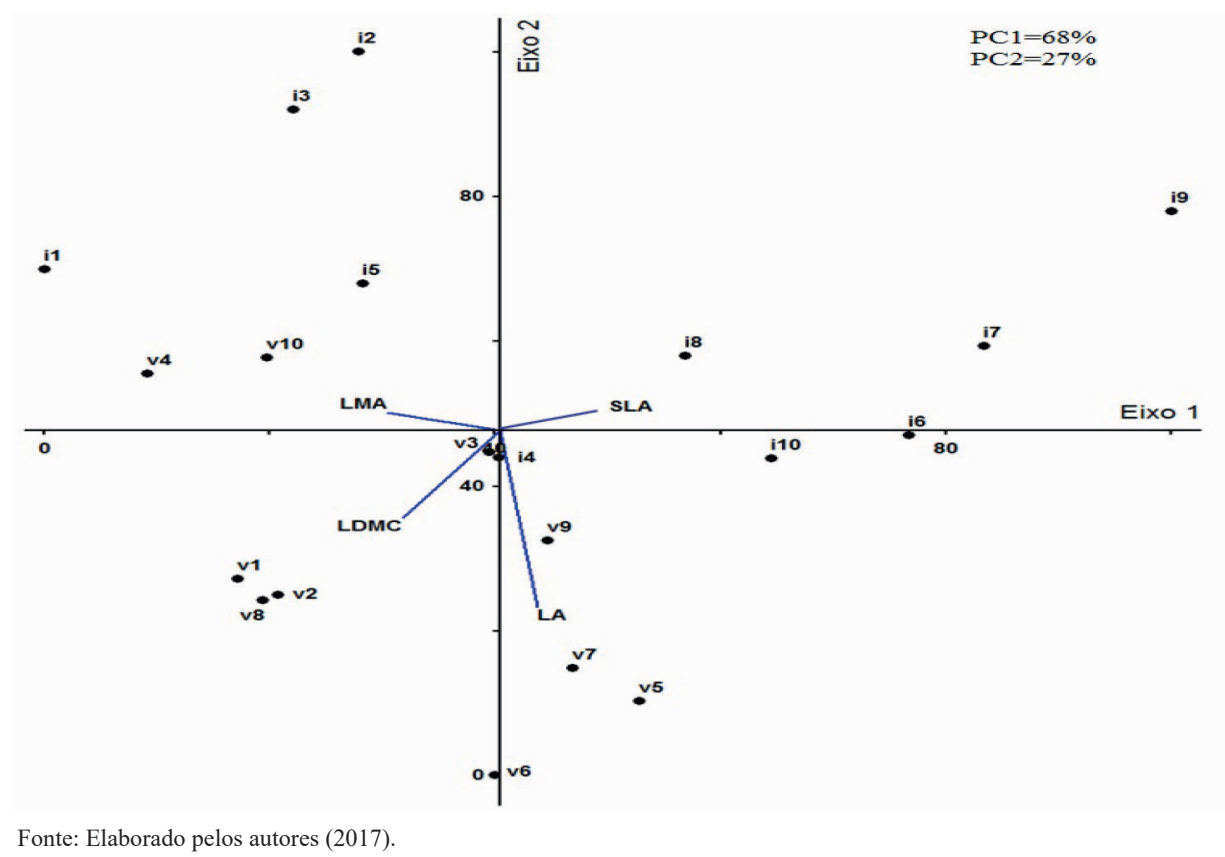

\section{Biometria das sementes}

Houve diferença significativa em todas as medições biométricas realizadas, sendo os valores maiores observados nas sementes de pitaica (S. polyphylla) da floresta de várzea em comparação as da floresta de igapó (Tabela 2).

Tabela 2. Valores biométricos obtidos nas medições de sementes de pitaica (Swartzia polyphylla) entre as florestas de igapó e várzea.

\begin{tabular}{|lcccc|}
\hline $\begin{array}{c}\text { Biometria da } \\
\text { semente }\end{array}$ & $\begin{array}{c}\text { Média e desvio } \\
\text { padrão várzea }\end{array}$ & $\begin{array}{c}\text { Média e desvio } \\
\text { padrão igapó }\end{array}$ & t & p \\
Peso fresco & $93,7(20,7)$ & $68,8(19,8)$ & $-8,39$ & 0,0001 \\
Peso seco & $52,7(14,7)$ & $40,2(15,0)$ & $-5,76$ & 0,0001 \\
Peso tegumento & $0,97(0,67)$ & $0,75(0,27)$ & -3 & 0,003 \\
Comprimento & $89,1(10,5)$ & $80,4(10,3)$ & $-5,7$ & 0,0001 \\
Espessura 1 & $44,1(4,7)$ & $40(4,85)$ & $-5,886$ & 0,0001 \\
Espessura 2 & $44,4(4,9)$ & $39(4,95)$ & $-7,457$ & 0,0001 \\
\hline
\end{tabular}

A biometria em sementes constitui um instrumento importante para detectar a variabilidade genética dentro de populações de uma mesma espécie e as relações entre essa variabilidade e os fatores ambientais, fornecendo importantes informações para a caracterização dos aspectos ecológicos como o tipo de dispersão, agentes dispersores e estabelecimento das plântulas (MACEDO et al., 2009). 


\section{Experimento 1 - Teste de germinação}

\section{Tempo de germinação}

Não houve diferença significativa do tempo de germinação das sementes entre as florestas de várzea e igapó ( $\mathrm{t}=-0,99 ; \mathrm{p}=0,32$, Figura $4 \mathrm{~A})$, sendo elas não correlacionadas com o peso fresco das sementes entre os tipos de floresta (Figura 4B).

Figura 4. Comparação do tempo de germinação das sementes de pitaica (Swartzia polyphylla) entre as florestas de igapó e várzea e com o peso fresco delas na Estação Científica Ferreira Penna, Caxiuanã, Pará.

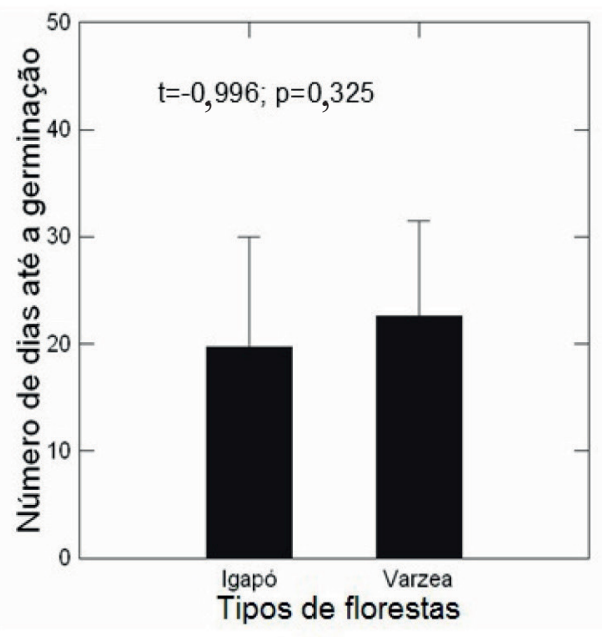

(A)

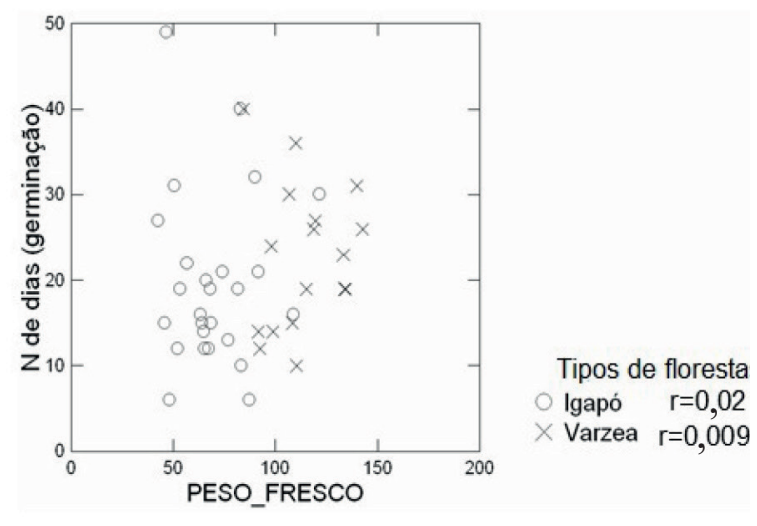

(B)

Essa questão pode sugerir que a espécie, assim como a maior parte das espécies tropicais, possui padrão de germinação intermediário como uma estratégia para fugir da predação, produzindo plântulas com maiores chances de escapar da herbivoria ${ }^{4}$ (NG, 1978). Além disso, o tegumento da semente aparentemente não influenciou nesse processo, pois sendo este mais pesado e consequentemente mais espesso nas sementes de várzea, poderia se esperar maior dificuldade para sua ruptura em relação às sementes de igapó demandando mais tempo para a geminação, o que não ocorreu (MALAVASI; MALAVASI, 2001).

\section{Taxa de germinação}

Houve diferença significativa na taxa de germinação de sementes (dos blocos) entre as florestas de igapó e várzea $(U=21,5 ; p=0,05)$, sendo esta maior nas florestas de igapó (Figura 5).

As taxas de germinação nos cinco blocos da floresta de várzea variaram de 33,3\% a 83,3\% $(X=57,6 \%)$, enquanto na floresta de igapó variou de 50\%-100\% (X=86,7\%).

A taxa de germinação maior nas sementes de igapó pode ser explicada pela maior ocorrência de fungos nas sementes de várzea verificada no estudo, consequência de um ambiente mais nutritivo (PAROLIN, 2010).

${ }^{4}$ Herbivoria: Relação interespecífica em que um animal alimenta-se de partes vivas dos vegetais. 
Figura 5. Mediana da taxa de germinação de sementes de pitaica (Swartzia polyphylla) entre as florestas de igapó e várzea da Estação Científica Ferreira Penna, Caxiuanã, Pará.

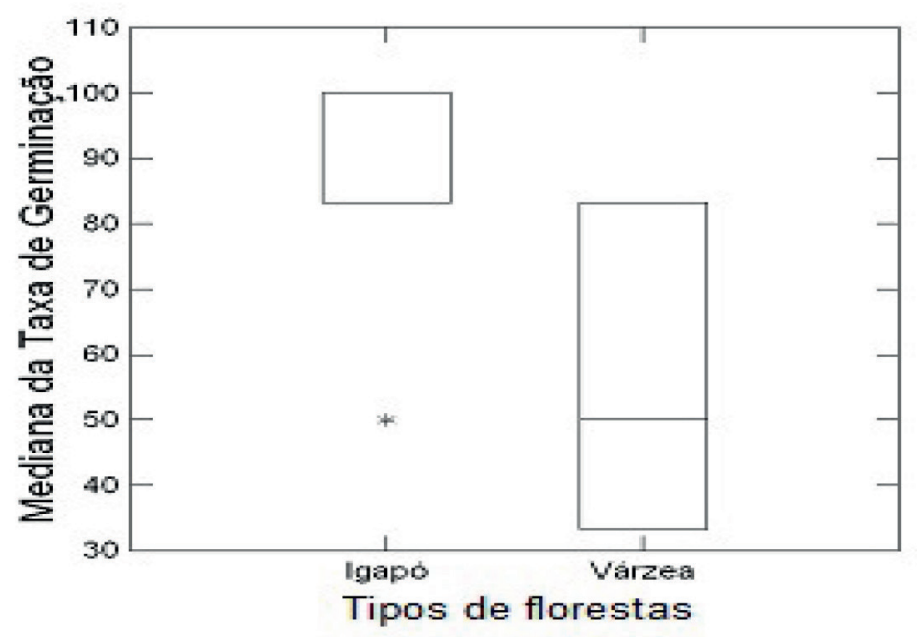

\section{Experimento 2 - Crescimento de plântulas}

As curvas de crescimento das sementes de pitaica (S. polyphylla) foram semelhantes entre os dois tipos de floresta (Figura 6A). Contudo, as sementes das florestas de várzea têm maior crescimento nos primeiros dias, devido à maior quantidade de endosperma (peso fresco). Entretanto, após 17 dias, o crescimento das plântulas é semelhante entre as duas florestas.

Não houve diferença significativa no tamanho final das plântulas entre as florestas de igapó e várzea $(\mathrm{t}=-0,12 ; \mathrm{p}=0,89$, Figura $6 \mathrm{~B})$.

Figura 6. Curva de crescimento e razão de crescimento das plântulas (A) germinadas de pitaica (Swartzia polyphylla) e tamanho final das plântulas entre os dois tipos de florestas da Estação Científica Ferreira Penna, Caxiuanã, Pará (B).

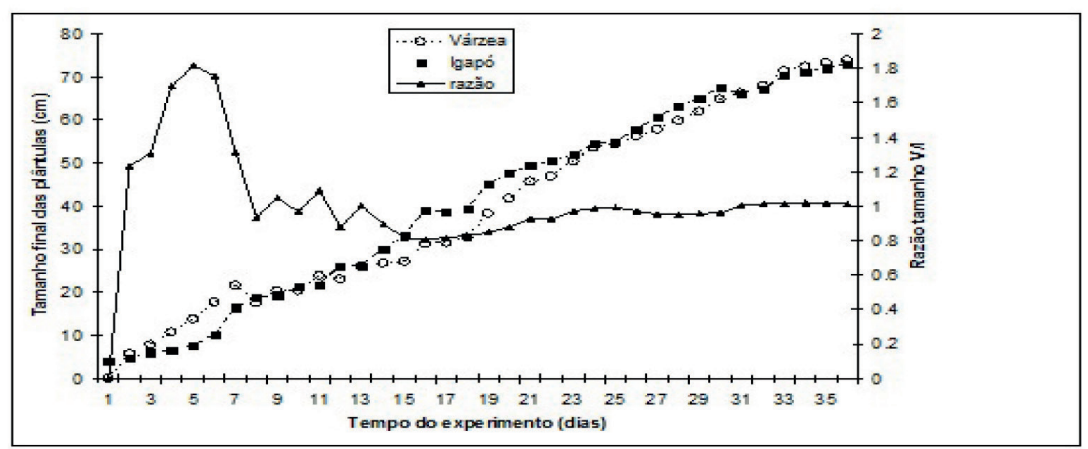

(A)

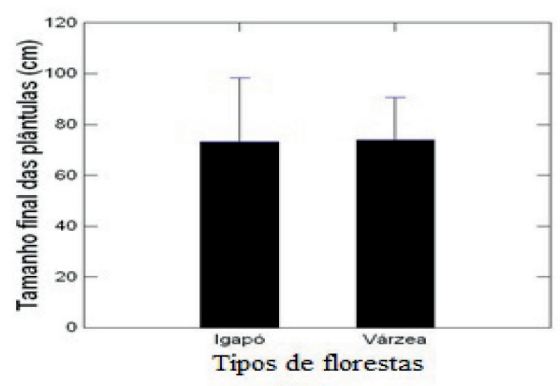

(B) 
Com relação ao crescimento inicial, as plântulas de várzea apresentaram maior crescimento, devido ao fato de possuírem mais reserva nutritiva em suas sementes (BUCKERIDGE et al., 2004; FERREIRA et al., 2009; BEWLEY et al., 2013), o que fica evidente no peso fresco e seco das sementes. Corroborando com essa afirmação temos o estudo de Moreira (1997), que verificou que espécies com maiores taxas de crescimento como S. polyphylla e Vatairea guianensis tinham as maiores sementes.

\section{Conclusão}

O estudo demonstrou que existem diferenças intraespecíficas dos indivíduos de pitaica $(S$. polyphylla $\mathrm{DC}$ ) em relação à densidade de indivíduos da regeneração natural e plantas estabelecidas com diâmetros até $10 \mathrm{~cm}$, na taxa de germinação de sementes e nos atributos funcionais foliares dos indivíduos entre as florestas inundadas de igapó e várzea, sendo isso resultante das diferenças de adaptações dos indivíduos às pressões impostas nos dois tipos de floresta.

Nas várzeas, mais ricas em nutrientes no solo, ocorre maior competição interespecífica resultando em maior investimento em atributos funcionais de crescimento, enquanto nos igapós, mais pobres em nutrientes, ocorre menor competição interespecífica e maior investimento em atributos funcionais conservativos.

Nos últimos anos houve um aumento do interesse da comunidade acadêmica, sejam eles ecólogos, botânicos ou agrônomos, para tornar conhecidos os mecanismos de propagação e sobrevivência de espécies florestais nativas do Brasil, tanto para o entendimento ecofisiológico, que possibilita compreender o comportamento das espécies em condições naturais, quanto para a produção de mudas. Assim, estudos sobre o comportamento germinativo de sementes e crescimento de plântulas tornam-se fundamentais para a compreensão de tais mecanismos, além de servirem como base para a silvicultura, conservação e manejo sustentável das populações de plantas. No entanto, ainda há carência de informações referentes às condições ideais de germinação. Isso pode ser verificado nas Regras para Análise de Sementes (BRASIL, 2009), nas quais são encontradas poucas prescrições para análise de sementes de espécies florestais.

\section{Referências}

ACKERLY, D. D.; CORNWELL, W. K. A trait-based approach to community assembly: partitioning of species trait values into within- and among-community components. Ecology Letters, v. 10, n. 2, p. 135145, Feb. 2007.

ARAÚJO, M. F.; VIEIRA, I. J. C.; BRAZ-FILHO, R.; MOTTA, O. V.; MATHIAS, L. Chemical constituents from Swartzia apetala Raddi var. glabra and evaluation of their antifungal activity against Candida spp. Revista Brasileira de Farmacognosia, v. 19, n. 2A, p. 366-369, Apr./June 2009.

AYRES, J. M. As matas de várzea do Mamirauá: médio Rio Solimões. 3. ed. Belém, PA: Sociedade Civil Mamirauá, 2006. 123 p. (Estudos do Mamirauá, v. 1).

BEWLEY, J. D.; BRADFORD, K. J.; HILHORST, H. W. M.; NONOGAKI, H. Seeds: physiology of development, germination and dormancy. 3. ed. New York: Springer, 2013. 392 p. 
BRASIL. Ministério do Meio Ambiente. Avaliação e identificação de áreas e ações prioritárias para a conservação, utilização sustentável e repartição dos benefícios da biodiversidade nos biomas brasileiros. Brasília, DF, 2002. 404 p.

BRASIL. Ministério da Agricultura, Pecuária e Abastecimento. Regras para análise de sementes. Brasília, 2009. 399 p.

BUCKERIDGE, M. S.; AIDAR, M. P.; SANTOS, H. P.; TINÉ, M. A. S. Acúmulo de Reservas. In: FERREIRA, A. G.; BORGHETTI, F. (Ed.). Germinação: do básico ao aplicado. Porto Alegre: Artmed, 2004. p. 31-50.

CORNWELL, W. K.; ACKERLY, D. D. Community assembly and shifts in plant trait distributions across an environmental gradient in coastal California. Ecological Monographs, v. 79, n. 1, p. 109-126, Feb. 2009.

COWAN, R. S. Swartzia (Leguminosae, Caesalpinioideae, Swartzieae). New York; London: Hafner Pub. Co., 1967. 228 p. (Flora neotropica, monograph, v. 1).

DUARTE, C. M. Methods in comparative functional ecology. In: PUGNAIRE, F. I.; VALLADARES, F. (Ed.). Handbook of functional plant ecology. New York: Marcel Dekker, 1999, p. 1-8.

FENNER, M.; THOMPSON, K. The ecology of seeds. Cambridge: Cambridge University Press, 2005. $250 \mathrm{p}$.

FERREIRA, C. S.; PIEDADE, M. T. F.; JUNK, W. J.; PAROLIN, P. Floodplain and upland populations of Amazonian Himatanthus sucuuba: effects of flooding on germination, seedling growth and mortality. Environmental and Experimental Botany, v. 60, n. 3, p. 477-483, July 2007.

FERREIRA, C. S.; PIEDADE, M. T. F.; FRANCO, A. C.; GONÇALVES, J. F. C.; JUNK, W. J. Adaptive strategies to tolerate prolonged flooding in seedlings of floodplain, upland populations of Himatanthus sucuuba, a Central Amazon tree. Aquatic Botany, v. 90, n. 3, p. 246-252, Apr. 2009.

FERREIRA, L. V.; SILVA, A. S.; ALMEIDA, S. S. Os tipos de vegetação de Caxiuanã. In: INSTITUTO CHICO MENDES DE CONSERVAÇÃO DA BIODIVERSIDADE - ICMBIO. Plano de manejo da Floresta Nacional de Caxiuanã. Brasília, DF, 2012. v. 1, p. 109-129.

GOULDING, M. The fishes and the forest: explorations in Amazonian Natural History. Berkeley: University of California Press, 1980. p. 1-280.

HENNING, F. A.; MERTZ, L. M.; JUNIOR, E. A. J.; MACHADO, R. D.; FISS, G.; ZIMMER, P. D. Composição química e mobilização de reservas em sementes de soja de alto e baixo vigor. Bragantia, v. 69, n. 3, p. 727-734, 2010.

HULSHOF, C. M.; SWENSON, N. G. Variation in leaf functional trait values within and across individuals and species: an example from a Costa Rican dry forest. Functional Ecology, v. 24, n. 1, p. 217-223, Feb. 2010.

JORDANO, P. Fruits and frugivory. In: FENNER, M. $(E d$.). Seeds: the ecology of regeneration in plant communities. 2. ed. Wallingford, UK: CABI Publ., 2000. p. 125-166.

JUNK, W. J. Ecology of the várzea floodplain of Amazonian white water rivers. In: SIOLI, H. (Ed.). The Amazon: limnology and landscape ecology of a mighty tropical river and its basin. Dordrecht: Dr. W.J. Junk Publishers, 1984. p. 215-243. (Monographiae Biologicae, v. 56). 
JUNK, W. J. Flood tolerance and tree distribution in Central Amazonian floodplains. In: HOLM-NIELSEN, L. B.; NIELSEN, I. C.; BALSLEV, H. (Ed.). Tropical forests: botanical dynamics, speciation and diversity. London: Academic Press, 1989. p. 47-64.

JUNK, W. J. Wetlands of tropical South America. In: WHIGHAM, D. F.; DYKYJOVÁ, D.; HEJNÝ, S. $(E d$.). Wetlands of the World I: inventory, ecology and management. Dordrecht: Springer, 1993. p. 679739. (Handbook of vegetation science, v. 15/2).

JUNK, W. J. (Ed). The Central Amazon Floodplain: ecology of a pulsing system. Berlin: Springer, 1997. 521 p. (Ecological Studies, v. 126).

KLINGE, H.; FURCH, K.; HARMS, E.; REVILLA, J. Foliar nutrient levels of native tree species from central Amazonia: I. Inundation forests (varzea, igapo). Amazoniana, v. 8, n. 1, p. 19-45, Jan. 1983.

KRAFT, N. J. B.; VALENCIA, R.; ACKERLY, D. D. Functional traits and nichebased tree community assembly in an Amazonian forest. Science, v. 322, n. 5901, p. 580-582, Oct. 2008.

KUBITZKI, K. The dispersal of forest plants. In: PRANCE, G. T.; LOVEJOY, T. E. (Ed.). Key environment: Amazonia. Oxford: Pergamon Press, 1985. p. 129-163. (Key Environment).

KUBITZKI, K.; ZIBURSKI, A. Seed dispersal in floodplain forests of Amazonia. Biotropica, v. 26, n. 1, p. 30-43, Mar. 1994.

LEISHMAN, M. R.; WRIGHT, I. J.; MOLES, A. T.; WESTOBY, M. The evolutionary ecology of seed size. In: FENNER, M. (Ed.). Seeds: the ecology of regeneration in plant communities. 2. ed. Wallingford: CABI Publishing, 2000. p. 31-57.

MACARTHUR, R. H.; LEVINS, R. The limiting similarity, convergence and divergence of coexisting species. The American Naturalist, v. 101, n. 921, p. 377-385, Sept./Oct. 1967.

MACEDO, M. C. D.; SCALON, S. D. P. Q.; SARI, A. P.; SCALON FILHO, H.; ROSA, Y. B. C. J.; ROBAINA, A. D. Biometry of fruit and seeds and germination of Magonia pubescens ST. Hil (SAPINDACEAE). Revista Brasileira de Sementes, v. 31, n. 2, p. 202-211, 2009.

MALAVASI, U. C.; MALAVASI, M. M. Influência do tamanho e do peso da semente na germinação e no estabelecimento de espécies de diferentes estágios da sucessão vegetal. Floresta e Ambiente, v. 8, n. 1, p. 211-215, 2001.

MANUAL técnico da vegetação brasileira. 2. ed. rev. ampl. Rio de Janeiro: IBGE, 2012. 274 p. (Manuais técnicos em geociências, n. 1).

MAY, R. M. Will a large complex system be stable?. Nature, v. 238, p. 413-414, Aug. 1972.

MELO, M. G. G.; MENDONÇA, M. S.; MENDES, A. M. S. Análise morfológica de sementes, germinação e plântulas de jatobá (Hymenaea intermedia Ducke var. adenotricha (Ducke) Lee \& Lang.) (Leguminosae-caesalpinioideae). Acta Amazônica, v. 34, n. 1, p. 9-14, 2004.

MICHAELS, H. J.; BENNER, B.; HARTGERINK, A. P.; LEE, T. D.; RICE, S.; WILLSON, M. F.; BERTIN, R. I. Seed size variation: magnitude, distribution, and ecological correlates. Evolution Ecology, v. 2, n. 2, p. 157-166, Apr. 1988.

MOEGENBURG, S. M. Sabal palmetto seed size: causes of variation, choices of predators, and consequences for seedlings. Oecologia, v. 106, n. 4, p. 539-543, June 1996.

MOEGENBURG, S. M. Spatial and temporal variation in hydrochory in Amazonian floodplain forest. Biotropica, v. 34, n. 4, p. 606-612, Dec. 2002. 
MONTEIRO, J. M.; ALBUQUERQUE, U. P.; ARAÚJO, E. L.; AMORIM, E. L. C. Taninos: uma abordagem da química à ecologia. Química Nova, v. 28, n. 5, p. 892-896, 2005.

MOREIRA, F. M. S. Nodulação e crescimento de 49 leguminosas arbóreas nativas da Amazônia em viveiro. Revista Brasileira de Ciência do Solo, v. 21, n. 4, p. 581-590, out. 1997.

NG, F. S. P. Strategies of establishment in Malayan forest trees. In: TOMLINSON, P. B.; ZIMMERMANN, M. H. (Ed.). Tropical trees as living systems. Cambridge: Cambridge University Press, 1978. p. 129-162.

NORDEN, N.; DAWS, M. I.; ANTOINE, C.; GONZALEZ, M. A.; GARWOOD, N. C.; CHAVE, J. The relationship between seed mass and mean time to germination for 1037 tree species across five tropical forests. Functional Ecology, v. 23, n. 1, p. 203-210, Feb. 2009.

PAROLIN, P. Seed mass in Amazonian floodplain forests with contrasting nutrient supplies. Journal of Tropical Ecology, v. 16, n. 3, p. 417-428, May 2000.

PAROLIN, P. Seed germination and early establishment of 12 tree species from nutrient-rich and nutrient-poor Central Amazonian floodplains. Aquatic Botany, v. 70, n. 2, p. 89-103, June 2001.

PAROLIN, P. Submergence tolerance vs. escape from submergence: two strategies of seedling establishment in Amazonian floodplains. Environmental and Experimental Botany, v. 48, n. 2, p. 177-186, Sept. 2002.

PAROLIN, P.; JUNK, W. J. The effect of submergence on seed germination in trees from Amazonian floodplains. Boletim do Museu Paraense Emílio Goeldi Série Botânica, v. 18, n. 1, p. 321-329, jan. 2002.

PAROLIN, P.; FERREIRA, L. V.; JUNK, W. J. Germination characteristics and establishment of trees from Central Amazonian flood plains. Tropical Ecology, v. 44, n. 2, p. 157-169, Jan. 2003.

PAROLIN, P.; DE SIMONE, O.; HAASE, K.; WALDHOFF, D.; ROTTENBERGER, S.; KUHN, U.; KESSELMEIER, J.; SCHMIDT, W.; PIEDADE, M. T. F.; JUNK, W. J. Central Amazon floodplain forests: tree survival in a pulsing system. The Botanical Review, v. 70, n. 3, p. 357-380, 2004.

PAROLIN, P. Flood-tolerant trees of Amazonian floodplains also tolerate drought. Invited paper. Pesquisas Botânica, v. 61, p. 7-38, June 2010.

PELAZZA, B. B.; SEGATO, S. V.; ROMANATO, F. N. Quebra de dormência em sementes de Adenanthera pavonina L. Nucleus, v. 8, n. 1, p. 305-314, abr. 2011.

PÉREZ-HARGUINDEGUY, N.; DÍAZ. S.; GARNIER, E.; LAVOREL, S.; POORTER, H.; JAUREGUIBERRY, P.; BRET-HARTE, M. S.; CORNWELL,W. K.; CRAINE, J. M.; GURVICH, D. E.; URCELAY, C.; VENEKLAAS, E. J.; REICH, P. B.; POORTER, L.; WRIGHT, I. J.; RAY, P.; ENRICO, L.; PAUSAS, J. G.; BUCHMANN, A. C. DE VOS, N.; FUNES, G.; QUÉTIER, F.; HODGSON, J. G.; THOMPSON, K.; MORGAN, H. D.; STEEGE, H. TER; HEIJDEN, M.G. A. VAN DER; SACK, L.; BLONDER, B.; POSCHLOD, P.; VAIERETTI, M. V.; CONTI, G.; STAVER, A. C.; AQUINO S.; CORNELISSEN J. H. C. New handbook for standardised measurement of plant functional traits worldwide. Australian Journal of Botany, v. 61, p. 167-234, Apr. 2013.

PHILIPSON, C. D.; DENT, D. H.; O’BRIEN, M. J.; CHAMAGNE, J.; DZULKIFLI, D.; NILUS, R.; PHILIPS, S.; REYNOLDS, G.; SANER, P.; HECTOR, A. A trait-based trade-off between growth and mortality: evidence from 15 tropical tree species using size-specific relative growth rates. Ecology and Evolution, v. 4, n. 18, p. 3675-3688, Sept. 2014. 
PICCININ, J.; RUIVO, M. L. Os solos da Floresta Nacional de Caxiuanã. In: INSTITUTO CHICO MENDES DE CONSERVAÇÃO DA BIODIVERSIDADE - ICMBIO. Plano de Manejo da Floresta Nacional de Caxiuanã. Brasília, DF, 2012. v. 1, p. 97-104.

POORTER, L.; BONGERS, F. Leaf traits are good predictors of plant performance across 53 rain forest species. Ecology, v. 87, n. 7, p. 1733-1743, July 2006.

POORTER, H.; NIINEMETS, Ü.; POORTER, L.; WRIGHT, I. J.; VILLAR, R. Causes and consequences of variation in leaf mass per area (LMA): a meta-analysis. New Phytologist, v. 182, n. 3, p. 565-588, May 2009.

PRADO JUNIOR, J. A.; VALE, V. S.; LOPES, S. F.; ARANTES, C.S.; OLIVEIRA, A. P.; SCHIAVINI, I. Impacts of disturbance intensity in functional traits patterns in understories of seasonal forests. Bioscience Journal, v. 30, n. 2, p. 901-911, Oct. 2014.

RASBAND, W. S. ImageJ. Maryland, EUA: Research Services Branch, National Institute of Mental Health, s.d. 1.44 p. Disponível em: http://imagej.nih.gov/ij/. Acesso em: 5 set. 2016.

REICH, P. B.; ELLSWORTH, D. S.; WALTERS, M. B.; VOSE, J. M.; GRESHAM, C.; VOLIN, J. C.; BOWMAN, W. D. Generality of leaf trait relationships: a test across six biomes. Ecology, v. 80, n. 6, p. 1955-1969, Sept. 1999.

REICH, P. B.; WALTERS, M. B.; ELLSWORTH, D. S. From tropics to tundra: global convergence in plant functioning. Proceedings of the National Academy of Sciences of the United States of America, v. 94, n. 25, p. 13730-13734, Dec. 1997.

REICH, P. B.; WRIGHT, L. J.; CARVENDER-BARES, J.; CRAINE, J. M.; OLEKSYN, J.; WESTOBY, M.; WALTERS, M. B. The evolution of plant functional variation: traits, spectra and strategies. International Journal of Plant Sciences, v. 164, n. 3, p. 143-164, 2003.

RIBEIRO, J. E. L. S.; HOPKINS, M. J. G.; VICENTINI, A.; SOTHERS, C. A.; COSTA, M. A. S.; BRITO, J. M.; SOUZA, M. A. D.; MARTINS, L. H. P.; LOHMANN, L. G.; ASSUNÇÃO, P. A. C. L.; PEREIRA, E. C.; SILVA, C. F.; MESQUITA, M. R.; PROCÓPIO, L. C. Flora da Reserva Ducke: guia de identificação das plantas vasculares de uma floresta de terra-firme na Amazônia Central. Manaus: INPA, 1999. $799 \mathrm{p}$.

SANCHEZ, C. S.; ROCHA, A. F. I.; PINHEIRO, M. L. B.; ANDRADE, C. H. S.; MONTE, F. J. Q. Brachyrachisina- Isoflavona inédita de Swartzia (Leguminosae). Acta Amazônica, v. 29, n. 3, p. 419-422, Sept. 1999.

SCARANO, F. R.; RIOS, R. I.; ESTEVES, F. A. Tree species richness, diversity and flooding regime: case studies of recuperation after anthropic impact in Brazilian flood-prone forests. International Journal of Ecology and Environmental Sciences, v. 24, n. 2, p. 223-235, June 1998.

SCARANO, F. R.; PEREIRA, T. S.; ROCAS, G. Seed germination during flotation and seedling growth of Carapa guianensis, a tree from flood-prone forests of the Amazon. Plant Ecology, v. 168, n. 2, p. 291296, Sept. 2003.

SERVIÇO FLORESTAL BRASILEIRO - SFB. Sistema Nacional de Informações Florestais - SNIF. Brasília, 2019. Disponível em: http://snif.florestal.gov.br/pt-br/. Acesso em: 14 jan. 2020.

SIOLI, H. Amazônia: fundamentos da ecologia da maior região de florestas tropicais. 3. ed. Petrópolis: Vozes, 1991. 69 p. 
SORIANO, D.; OROZCO-SEGOVIA, A.; MÁRQUEZ-GUZMÁN, J.; KITAJIMA, K.; BUEN, A. G.; HUANTE, P. Seed reserve composition in 19 tree species of a tropical deciduous forest in Mexico and its relationship to seed germination and seedling growth. Annals of Botany, v. 107, n. 6, p. 939-951, Mar. 2011.

SOUZA, B. C.; OLIVEIRA, S. R.; ARAÚJO, F. S.; LIMA, A. L. A.; RODAL, M. I. N. Divergências funcionais e estratégias de resistência à seca entre espécies decíduas e sempre verdes tropicais. Rodriguésia, v. 66 , n. 1, p. 21-32, mar. 2015.

SWENSON, N. G.; ENQUIST, B. J. Ecological and evolutionary determinants of a key plant functional trait: wood density and its community-wide variation across latitude and elevation. American Journal of Botany, v. 94, n. 3, p. 451-459, Mar. 2007.

VIOLLE, C.; JIANG, L. Towards a trait-based quantification of species niche. Journal of Plant Ecology, v. 2, n. 2, p. 87-93, May 2009.

VIOLLE, C.; NAVAS, M. L.; VILE, D.; KAZAKOU, E.; FORTUNEL, C.; HUMMEL, L.; GARNIER, E. Let the concept of trait be functional! Oikos, v. 116, n. 5, p. 882-892, Jan. 2007.

WESTOBY, M.; JURADO, E.; LEISHMAN, M. Comparative evolutionary ecology of seed size. Trends in Ecology and Evolution, v. 7, n. 11, p. 368-372, Nov. 1992.

WILLIAMSON, B. G.; COSTA, F.; VERA, M. V. C. Dispersal of Amazonian trees: hydrochory in Swartzia polyphylla. Biotropica, v. 31, n. 3, p. 460-465, Sept. 1999.

WITTMANN, A. O.; PIEDADE, M. T. F.; PAROLIN, P.; WITTMANN, F. Germination in four low-várzea tree species of Central Amazonia. Aquatic Botany, v. 86, n. 3, p. 197-203, Apr. 2007.

WITTMANN, F.; ANHUF, D.; JUNK, W. J. Tree species distribution and community structure of Central Amazonian varzea forests by remote sensing techniques. Journal of Tropical Ecology, v. 18, n. 6, p. 805820, Nov. 2002.

WRIGHT, I. J; CANNON, K. Relationships between leaf lifespan and structural defences in a low nutrient, sclerophyll flora. Funcional Ecology, v. 15, n. 3, p. 351-359, June 2001.

WRIGHT, S. J.; KITAJIMA, K.; KRAFT, N. J. B.; REICH, P. B.; WRIGHT, I. J.; BUNKER, D. E.; CONDIT, R.; DALLING, J. W.; DAVIES, S. J.; DÍAZ, S.; ENGELBRECHT, B. M. J.; HARMS, K. E.; HUBBELL, S. P.; MARKS, C. O.; RUIZ-JAEN, M. C.; SALVADOR, C. M.; ZANNE, A. E. Functional traits and the growth-mortality tradeoff in tropical trees. Ecology, v. 91, n. 2, p. 3664-3674, Dec. 2010.

ZAR, J. H. Bioestatistical analysis. 5. ed. New Jersey: Prentice Hall, 2010. 944 p. 
